\title{
De las aulas a los ambientes naturales... Conservamos nuestro patrimonio natural
}

Verónica L. Romero ${ }^{1}$, Martin M. Kowalewski² ${ }^{2}$ Jorge A. Céspedez ${ }^{3}$, Angelina M. Godoy ${ }^{4}$, César A. Obregón ${ }^{4}$, Bárbara G. Romero ${ }^{4}$ Patricia M. Fernández ${ }^{4}$, Rocío A. Alegre ${ }^{5}$, Rodrigo Bay ${ }^{5}$, Mauricio A. Landi ${ }^{5}$, Joaquín Mayer ${ }^{5}$, Daniela Pucheta ${ }^{5}$, Romina F. Quijano ${ }^{5}$, Marta. I. G. Romero $\left.{ }^{(*}\right)$

\section{Resumen}

El presente trabajo es resultado del proyecto de extensión UNNE res. 109/18 C.S., en el cual participaron integrantes de la FaCENA (UNNE), EBCo (MACNCONICET), docentes y estudiantes de distintos niveles educativos de la provincia de Corrientes, guardaparques del Parque Pcial San Cayetano (PPSC, San Cayetano, Corrientes) y guías del Museo de Ciencias Naturales Amado Bonpland y de Casa Iberá (Corrientes, Corrientes). El objetivo principal fue fortalecer valores ambientales relacionados a temas de biodiversidad y conservación, principalmente mamíferos y sus ambientes naturales. Entre ellos, se trató aquellas especies bajo protección como los monumentos naturales de la provincia (aguará guazú, lobito de río, ciervo de los pantanos, venados de las pampas y oso hormiguero), y otras de importancia ecológica, cultural y/o económica como monos carayá, guazunchos, zorros, carpinchos y tatúes. Esta experiencia fue un punto de partida para la integración didáctica entre los conocimientos científicos generados en la EBCo y la UNNE y los contenidos del Diseño Curricular de Corrientes, siendo la metodología talleres de educación no formal en las aulas y visitas guiadas al PPSC, Museo

\footnotetext{
${ }^{1}$ Doctora de la UNNE en Biología, Directora Proyecto Extensión UNNE res. 109/18 C. S., Celular: +54-379-4054663, lorenromer@yahoo.com.ar.

${ }^{2}$ Doctor en Antropología, Dir. de la Estación Biológica Corrientes, Celular: +54-379-4245581, martinkow@gmail.com. ${ }^{3}$ Lic. en Zoología, Co-Director Proyecto Extensión UNNE res. 109/18 C. S., Celular: +54-379-4,

${ }^{4}$ Integrantes del área de Educación Ambiental, Estación Biológica Corrientes, Celular: +54-379-4245581, edu.ambientalebco@gmail.com

${ }^{5}$ Estudiantes universitarios de la Facultad de Ciencias Exactas y Naturales y Agrimensura, integrantes de Proyecto de Extensión UNNE res. 109/18 C. S., Celular: +54-379-4054663, lorenromer@yahoo.com.ar.

(*) Facultad de Ciencias Exactas y Naturales y Agrimensura, Avenida Libertad 5470, CP: 3400, ciudad de Corrientes
} 
de Cs. Naturales Bonpland y/o Casa Iberá. Esta interacción permitió a la comunidad involucrada, el desarrollo de habilidades, actitudes y conocimientos en espacios generados para favorecer el reconocimiento, la reflexión, la valoración, el respeto de su $\mathrm{Pa}$ trimonio Natural como un elemento clave hacia una mejor calidad de vida a futuro.

Palabras clave: Monumentos Naturales, Parque Provincial San Cayetano, Educación Ambiental.

\section{Introducción}

En el marco de proyectos de educación ambiental que forman parte de la Estación Biológica Corrientes (EBCo), sede del Museo Argentino de Ciencias Naturales Bernardino Rivadavia (MACN-CONICET) y de trabajos de investigación de grado y doctorales conjunta con la Facultad de Ciencias Exactas y Naturales y Agrimensura (FaCENA, UNNE), surgió la necesidad de trabajar sobre los efectos que causan la conversión de bosques nativos a sistemas agrícolas ganaderos siendo un problema medioambiental global (Hansen et al. 2013), el cual fomenta el cambio climático (Achard et al. 2014), y resulta en la pérdida de especies y procesos ecológicos, claves para el mantenimiento de la integridad de los ecosistemas (Melo et al. 2013, Laurance et al. 2014). En Argentina, los ambientes naturales han sufrido importantes modificaciones debido a la expansión de las fronteras agropecuarias, agroindustriales y urbanas, y amenaza a un gran número de especies de plantas y animales, entre ellos los primates como los carayás (Alouatta caraya), los cuales pasaron de especies no amenazadas (Díaz y Ojeda 2000) a vulnerable en un lapso de 10 años (Agostini et al. 2012). En este sentido, se considera fundamental la participación de la Universidad. Esta institución mediante un proyecto de extensión con carácter educativo-ambiental tiene la capacidad de articular distintos actores sociales. $Y$ en este sentido, desde una perspectiva sustentable, los recursos son bienes que deben ser disfrutados no sólo por las generaciones presentes, sino también por las que aún no han nacido (Flores 2016). Por lo tanto, los recursos naturales de Corrientes son bienes, que la comunidad debe valorar, tanto para garantizar la protección y conservación de su flora, fauna y sus paisajes y realizar un papel multiplicador hacia sus familias.

Consecuentemente, la implementación del proyecto de extensión aprobado por res. 109/18 C. S., con características marcadamente educativo-ambiental, fue imprescindible para aportar a la conservación de los recursos naturales de la provincia. Este recurso natural -que fue tratado desde su génesis en talleres específicos- estuvo representado por la comunidad de mamíferos silvestres, cuyas presencias y características biológicas, ecológicas, comportamentales y epidemiológicas representan el estado de salud de los paisajes naturales correntinos. Así, el desarrollo de encuentros educativos fueron fundamentales al generar propuestas participativas, y evitar que se desdoblen esfuerzos humanos, económicos, capacidades y conocimientos (Soler et al. 2005). Las acciones y actividades llevadas a cabo por 
integrantes de la $\mathrm{EBCo}$ (MACN, CONICET) y de la FaCENA (UNNE), buscaron relacionar las comunidades educativas de distintas localidades de Corrientes (Capital, Riachuelo, San Luis del Palmar) con el Parque Provincial San Cayetano (San Cayetano, Corrientes) y otras instituciones como el Museo de Ciencias Naturales Amado Bonpland y Casa Iberá. De este modo, el objetivo principal fue favorecer el fortalecimiento de valores ambientales y la integración didáctica entre los conocimientos científicos y los contenidos del Diseño curricular de Corrientes; promover la protección y conservación de los ambientes naturales a través de la valorización de los recursos de flora, fauna y de paisaje, como así también, sensibilizar a la comunidad educativa sobre las relaciones entre las personas y los ambientes, y las consecuencias de las acciones sobre la mastofauna local.

\section{Metodología}

En este proyecto de extensión, los conocimientos teóricos-prácticos se basaron en varios años de experiencia de investigación con especies de mamíferos y/o sus ambientes, desde el equipo de dirección de este proyecto (FaCENA, UNNE) como la coordinación general (EBCo, CONICET) e integrantes del GEM (Grupo de Estudios de Mamíferos, $\mathrm{EBC}$ ), y del área de Educación Ambiental de la EBCo y de conservación (EBCo, CONICET y Dirección de Parques y Reserva de la provincia de Corrientes). La experiencia en investigación, educación ambiental y conservación hizo que el equipo de trabajo es- tuviera capacitado para iniciar un proceso de articulación e integración, mediante la participación de docentes y estudiantes de cinco instituciones: 1) 85 alumnos del nivel inicial, escuela jardín de infantes $\mathrm{N}^{\circ}$ 34 (Laguna Brava, Corrientes); 2) 30 estudiantes de poli-grado, escuela primaria $\mathrm{N}^{\circ}$ 784 (Paraje Pontón, San Luis del Palmar); 3) 28 estudiantes de sexto año, colegio secundario Dr. Luis F. Leloir (Ciudad de Corrientes); 4) 20 estudiantes de sexto año, colegio secundario Cautivas Correntinas (Pirayuí, Corrientes); 5) 69 estudiantes de primer año, colegio secundario San Benito (Ciudad de Corrientes).

Las especies consideradas en el proyecto fueron las que se encuentran bajo protección como Monumentos Naturales de Corrientes (decreto $\mathrm{N}^{\circ}$ 1555/92): el aguará guazú (Chrysocyon bracbyurus), lobito de río (Lontra longicaudis), ciervo de los Pantanos (Blastocerus dichotomus), venados de la pampa (Ozotoceros bezoarticus) y el oso hormiguero (Myrmecophaga tridactyla). Además, otras especies de carnívoros de importancia ecológica como zorros de mediano porte (Cerdocyon thous y Lycalopex gymnocercus), herbívoros cuyo estado de conservación es vulnerable como los monos carayás (Alouatta caraya), y otros con un alto valor alimenticio, comercial y/o presa de actividades como la cacería entre ellos los carpinchos (Hydrochoerus bydrochaeris), los guazunchos (Mazama gouazoubira) y los tatúes (Euphractus sexcinctus, Dasypus novemcinctus). La posibilidad de cambiar la percepción de los carnívoros, como otros mamíferos, es muy importante y debe ge- 
nerarse desde temprana edad, y en distintos niveles educativos.

De esta manera, las acciones y actividades contempladas en esta propuesta, en parte buscó relacionar a las comunidades educativas propuestas como "co-participantes", vincular la teoría con la experiencia práctica de campo al visitar el Parque Provincial San Cayetano (PPSC, San Cayetano, Corrientes), el Museo de Ciencias Naturales Amado Bonpland y Casa Iberá, y favorecer el contacto directo con el medio natural y sus elementos, promoviendo el respeto $\mathrm{y}$ aprecio por los valores de las áreas Naturales (Programa Educativo Ambiental EBCo, 2009, 2018).

El proyecto tuvo dos etapas: la 1ra etapa) consistió en la implementación de talleres específicos por cada institución escolar ("Los secretos de nuestros ambientes naturales", "¿Mamíferos en Corrientes?... conocer para conservar"); y, la 2da etapa) visitas guiadas en el sendero de interpretación ambiental del PPSC, Museo de Ciencias Naturales Bonpland y/o Casa Iberá. En los talleres, las actividades propusieron un trabajo articulador e interdisciplinar de contenidos de la currícula escolar de cada nivel (inicial, primario, secundario) utilizando estrategias de educación no formal. Este tipo de propuesta, promueve un manejo del grupo innovador y la enseñanza mediante actividades originales, lúdicas y recreativas. En las visitas guiadas se hizo foco en el sistema ambiental local, que permitió afianzar valores, tomar consciencia del lugar donde se vive, promover la cooperación y el trabajo en equipo. Algunas temáticas relacionadas con la conservación de mamíferos se problematizaron, haciendo hincapié en las acciones del Hombre y las consecuencias en las dimensiones social-ambiental-económica.

Los encuentros fueron programados según la situación particular de cada institución. Los talleres específicos fueron desarrollados en el segundo trimestre escolar (agosto-septiembre). Y, las visitas guiadas en el tercer trimestre (octubre-noviembre), respetando tiempos y permisos para movilizar a los estudiantes y docentes al área protegida, museo o Casa Iberá. Cada encuentro tuvo momentos de preparación previa para la adecuación pedagógica con los integrantes del equipo de proyecto, particularmente con los estudiantes universitarios voluntarios de la FaCENA. Junto a ellos se realizó la preparación de tareas o actividades a desarrollar y se elaboraron materiales didácticos para los talleres áulicos. En todos los encuentros, las actividades de lecto-escritura con lecturas motivadoras, de reflexión, basados en cuentos y leyendas de origen popular, videos cortos para mostrar realidades que impactan sobre los ambientes naturales, fueron puntos centrales que permitieron revalorizar nuestra flora y fauna, los paisajes y la identidad territorial.

\section{Resultados y Conclusiones}

Las actividades expuestas han sido destinadas a cinco instituciones escolares, una escuela de nivel inicial, una escuela de nivel primario (rural y poligrado) y tres colegios secundarios de distintos puntos de Corrientes, siendo un total de 232 estudiantes y 17 docentes responsables de cursos. En total, 
durante 2018, se efectuaron siete talleres adaptados al nivel de cada institución, los tiempos y espacios físicos de cada establecimiento sumado a las condiciones meteorológicas (dos encuentros en el nivel inicial, un encuentro en la escuela primaria, dos encuentros en el colegio Leloir, un taller en el aula de la EBCo para el colegio Cautivas Correntinas y San Benito), y dos visitas al PPSC (colegio Cautivas Correntinas, San Benito), una visita al museo Bonpland y una visita a Casa Iberá (colegio Leloir). En el caso del nivel inicial y primario, las visitas guiadas no han sido por el momento llevadas a cabo, entre las razones: 1) tiempos en los permisos, 2) estado del tiempo (precipitación, alerta de inundación en el PPSC, altas temperaturas, condiciones del sendero de interpretación), 3) disponibilidad de transporte. Sin embargo, se han efectuado otras actividades importantes como reuniones de equipo, un taller informativo a cargo de la Dra. Verónica L. Romero y el Lic. César A. Obregón, una capacitación sobre Educación no formal a cargo del Dr. Martín M. Kowalewski, y un taller en el PPSC para los estudiantes voluntarios a cargo de las guías intérpretes ambientales Patricia M. Fernández y Bárbara G. Romero.

Entre los resultados esperados se logró la integración de los temas tratados en cada taller específico con en la visitas guiadas. $Y$ en el caso de las instituciones que no han podido concretar la visita, se logró realizar uno o dos talleres con actividades que estimulen los sentidos de los niños del jardín y la primaria. En estos espacios se logró aprender sobre las especies de mamíferos de interés, sus características corporales, comportamientos e importancia. Todas las actividades lograron el interés y la promoción de la protección y conservación de los ambientes naturales a través de la valorización de los recursos de flora, fauna y pasajísticos de Corrientes. Principalmente aquellos afectados por infraestructuras que actualmente generaron un impacto negativo en la cuenca del río Riachuelo (Riachuelo, Corrientes).

A futuro, aa aplicación de las actividades de los talleres específicos y las visitas guiadas durante el ciclo 2018 por cada docente responsable del curso, permitió formar un nexo que hará posible la continuidad de trabajo de proyectos similares con estos co-participantes propuestos y otras instituciones interesadas en las actividades ofrecidas.

En parte, el proyecto cumplió con su objetivo principal que fue transferir aquellos conocimientos científicos generados por la EBCo y la FaCENA, junto a aquellos proporcionados por el Museo Bonpland y Casa Iberá hacia las instituciones escolares. Dicha transferencia didáctica se realizó de una manera distinta y original desde las aulas a los ambientes naturales con la meta de valorar nuestro Patrimonio Natural.

Como toda experiencia de educación ambiental se apuntó a lograr un mundo más equitativo y participativo, y es más consciente de la necesidad de conservar el patrimonio cultural, social, humano y ecológico (Rose y Bridgewater 2003). Por lo tanto, la trascendencia de este y otros pro- 
yectos educativos se logrará a través de procesos que tengan en cuenta la génesis de los recursos, el conocimiento teórico-práctico orientado a la conservación y protección de estos recursos naturales, siendo preciso

\section{Bibliografía}

ACHARD F, et al. 2014. Determination of tropical deforestation rates and related carbon losses from 1990 to 2010. Global Change Biology, 20: 2540-2554.

AGOSTINI I, et al. 2012. Orden Primates. Pp. 81- 86. In: RA OJEDA, V CHILLO y GB DIAZ ISENRATH (eds.), Libro rojo de mamíferos amenazados de la Argentina. Sociedad Argentina para el Estudio de los Mamíferos.

DIAZ GB y R OJEDA. 2000. Libro Rojo. Mamíferos amenazados de la Argentina. SAREM. Buenos Aires.

FLORES, RC. 2016. Introducción. Pp.: 4-8. En: Flores, CR (ed.), Proyectos Educativos Sustentables, Instituto Pedagógico de Estudios de Posgrado, México.

HANSEN MC, et al. 2013. High-Resolution Global Maps of 21st-Century Forest Cover Change. Science: 850-853.

LAURANCE WF, et al. 2013. Agricultural expansion and its impacts on tropical nature. Trends in ecology and evolution, 29: 1-10.

MELO FPL, et al. 2013. On the hope for

\section{Agradecimientos}

A todos los directivos, docentes y estudiantes de la escuela jardín de infantes $\mathrm{N}^{\circ} 34$ (Laguna Brava, Corrientes), escuela educar para estimular transformaciones en los sistemas de pensamiento, cuya toma de decisiones impulse la participación pública en los problemas globales (Mendoza Flores 2016), regionales y locales.

biodiversity-friendly tropical landscapes. Trends in ecology and evolution, 28, 462-8. MENDOZA FLORES, N. 2016. Hablar de Sustentabilidad. Pp.: 2-3. En: Flores, CR (ed.), Proyectos Educativos Sustentables, Instituto Pedagógico de Estudios de Posgrado, México.

ROSE OH y P BRIDGEWATER. 2003. Se necesitan nuevos enfoques para la educación ambiental y la sensibilización del público. Perspectivas: revista trimestral de educación comparada, 27 (33): 20-32.

SOLER L, JM CARENTON, C CACERES, P PEREZ, A FLEITAS, A TABOADA, G MILLER y R GONZALEZ. 2005. La conservación de los carnívoros silvestres del Nordeste Argentino a través de la participación de los pobladores locales. Imagen y respuestas de los lugareños rurales hacia los predadores. MEMORIAS: Manejo de Fauna silvestre en Amazonia y Latinoamérica: 634-643.

primaria No 784 (Paraje Pontón, San Luis del Palmar), colegio secundario Dr. Luis F. Leloir (Ciudad de Corrientes) colegio se- 
cundario Cautivas Correntinas (Pirayuí, Prof. Carolina Blanco (Colegio Leloir), Corrientes) y colegio secundario San Be- Prof. Romina Sclippa (Colegio San Benito) nito (Ciudad de Corrientes) por participar y Prof. Milton Correa (Colegio Cautivas del proyecto de extensión (res. 109/18 C. Correntinas). A cada uno de los estudiantes S.), y permitirnos realizar los distintos encuentros y compartir con nosotros una gran experiencia con sus estudiantes. Particularmente, a las directoras Mariel Lirussi junto a su equipo docente del JIN $\mathrm{N}^{\circ} 34$ y la directora Mabel Miranda y su equipo docente de la escuela $\mathrm{N}^{\circ} 784$. A los docentes responsables de cursos de los colegios secundarios niñas/os y jóvenes participantes por jugar y aprender sobre los mamíferos y sus ambientes de nuestra región. Y, especialmente a los guías del Museo de Ciencias Naturales Amado Bonpland y Casa Iberá, y los guardaparques del Parque Provincial San Cayetano, por recibirnos y poner a disposición su tan cálida atención e instalaciones.

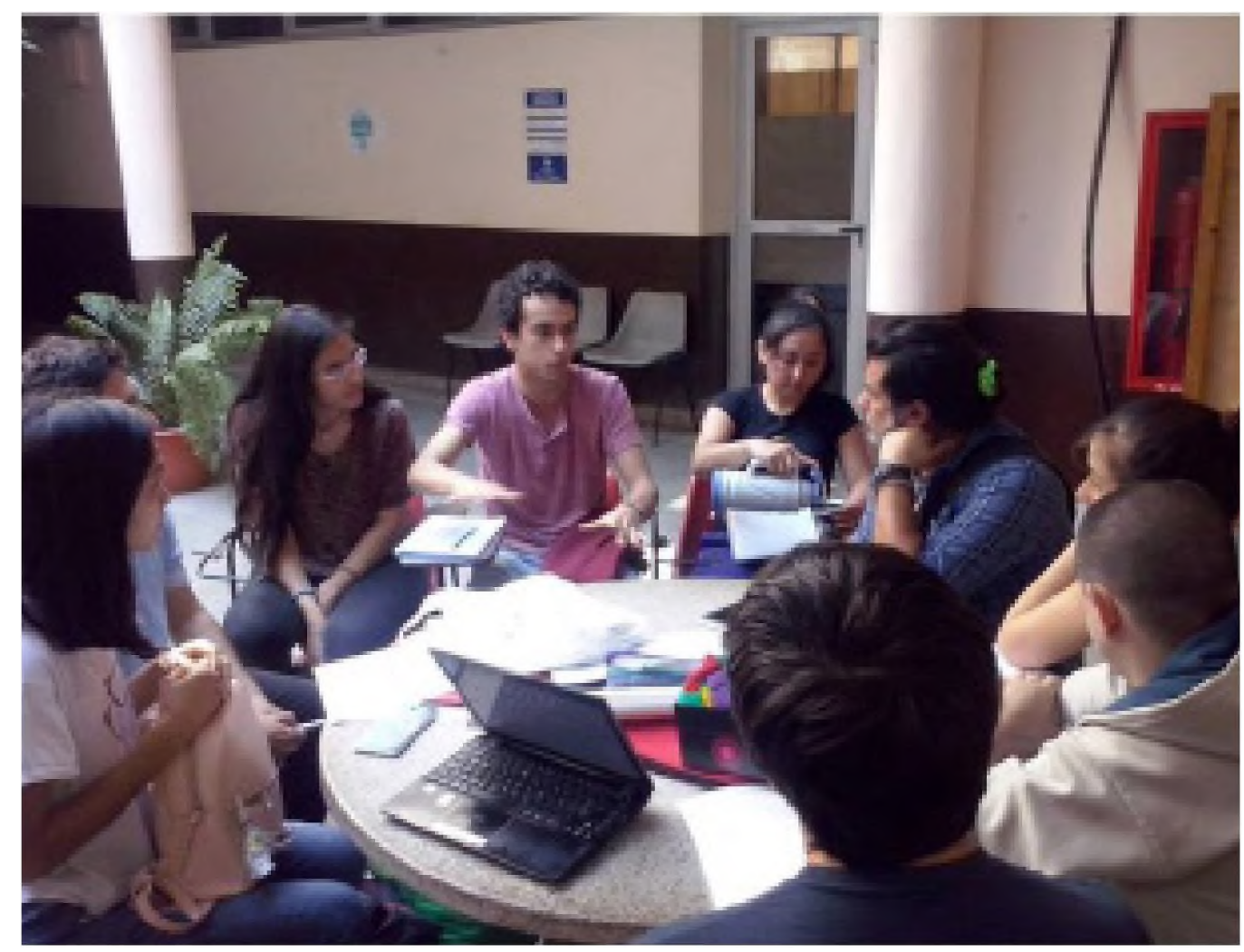

Figura 1: Reuniones de equipo entre integrantes del proyecto de extensión UNNE que complementaron a los talleres informativos y capacitación interna sobre Educación no formal e interpretación ambiental en el Parque Pcial San Cayetano (San Cayetano, Corrientes). 


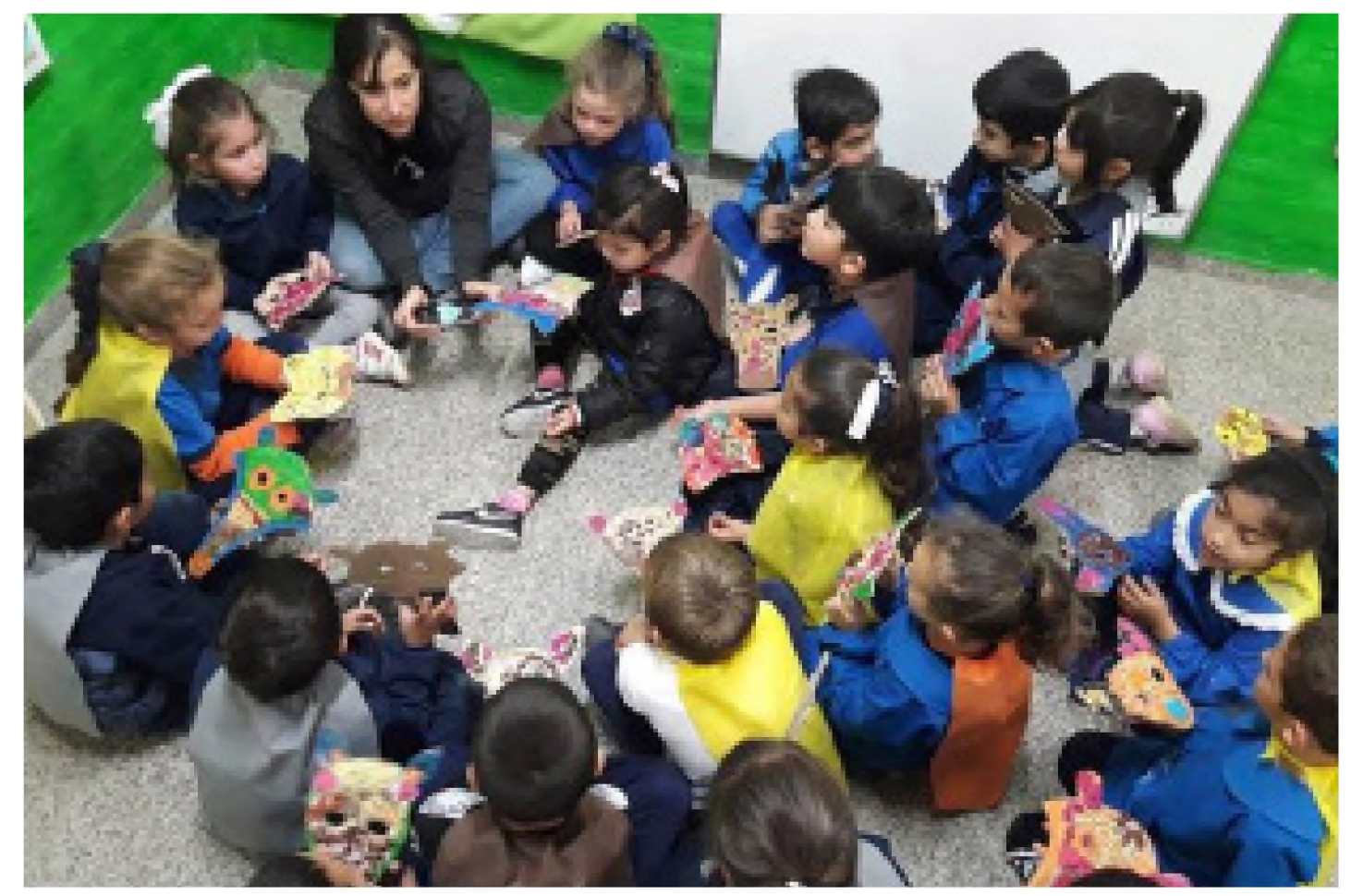

Figura 2: Talleres sobre mamiferos nativos y sus ambientes. Se ilustra el taller efectuado en el Jardin de Infantes $N^{\circ} 34$, Laguna Brava, Corrientes.

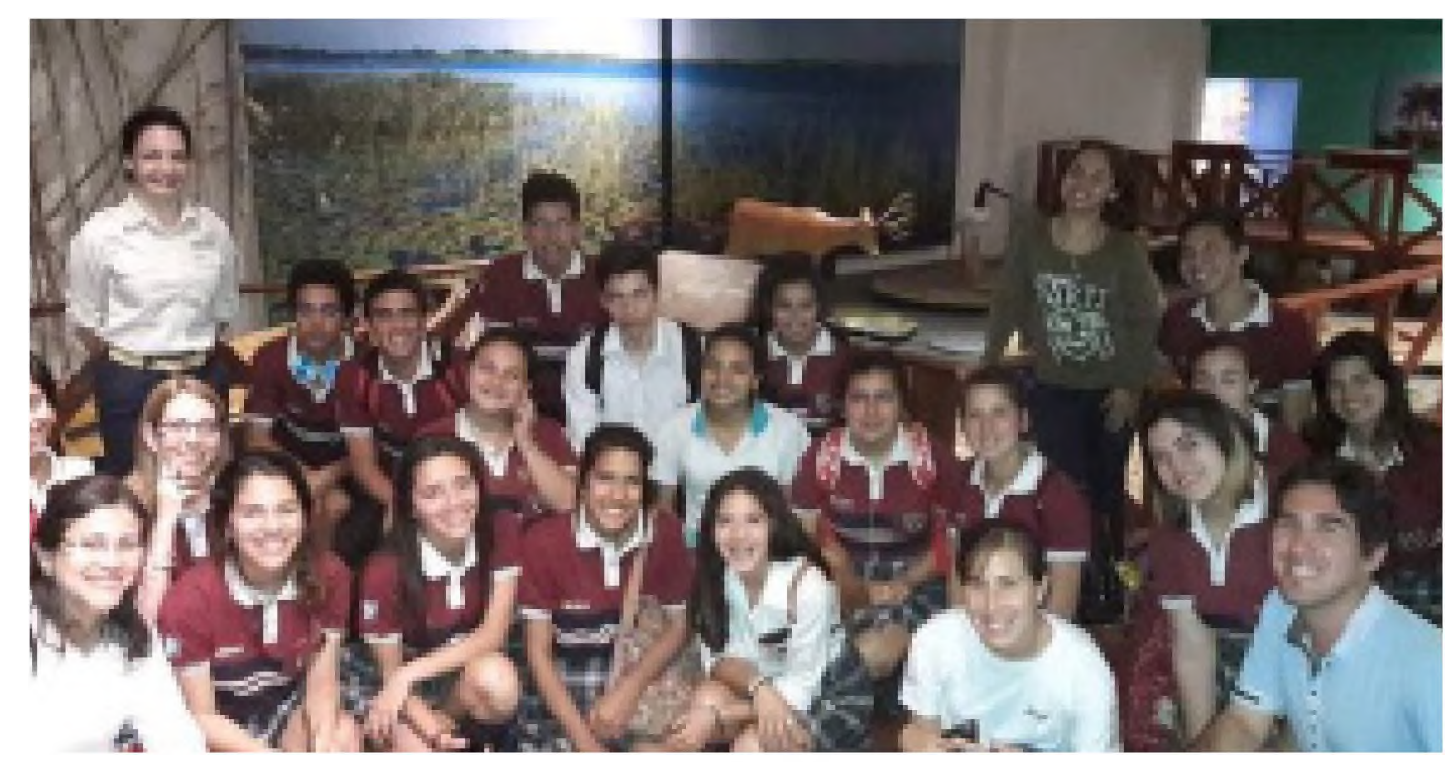

Figura 3: Visitas guiadas en Casa Iberá junto al Colegio Leloir, Corrientes. 